\title{
PROCESSAMENTO E CARACTERIZAÇÃO DE NÚCLEO DE FERRO PURO OBTIDO POR METALURGIA DO PÓ PARA APLICAÇÃO COMO CAPTADOR EM ENERGY HARVESTING
}

\author{
D. A. VIEIRA ${ }^{1 *}$, M. P. SANTOS ${ }^{1}$, D. S. G. LIMA ${ }^{1}$, Y. P. M. RODRIGUEZ ${ }^{1}$, C. P. SOUZA ${ }^{1}$ e T. O. MORAES JR. ${ }^{2}$ \\ ${ }^{1}$ Universidade Federal da Paraíba - UFPB \\ ${ }^{2}$ Universidade Federal de Campina Grande - UFCG \\ debora.vieira@cear.ufpb.br*
}

Artigo submetido em dezembro/2014 e aceito em maio/2015

DOI: $10.15628 /$ holos.2015.2655

\section{RESUMO}

Nos últimos anos, o termo colheita de energia tem sido utilizada em uma grande classe de aplicações, cuja principal função é a recuperação da energia disponível a partir de fontes, tais como, mecânica, solar, térmica, entre outras, que de outro modo seria desperdiçada. Nesse sentido, o objetivo deste estudo é o desenvolvimento e caracterização de um núcleo de pó de ferro obtido por moagem de alta energia, bem como a utilização desse núcleo como captador em um processo de colheita de energia por indução no intuito de observar a sua capacidade de colher energia. O ferro foi caracterizado por difração de raios $X$ e microscopia eletrônica de varredura e realizados testes de bancada para avaliar a quantidade de energia colhida do captador desenvolvido. A análise estrutural mostrou que o pó obtido é monofásico, sem presença de impurezas. As micrografias apresentam característica homogênea, com pouca porosidade. As características magnéticas do material obtido o classificam como material ferromagnético mole, devido ao estreito laço de histerese. Nos testes para captação de energia, o núcleo apresentou resultados satisfatórios, pois foi capaz de fornecer um nível máximo de potência de $0.41 \mathrm{~mW}$ quando submetido a uma corrente elétrica em seu enrolamento primário de $15 \mathrm{~A}$.

PALAVRAS-CHAVE: Ferro, colheita de energia, indução magnética, metalurgia do pó.

\section{PROCESSING AND CHARACTERISTICS OF IRON PURE CORE OBTAINED BY POWDER METALLURGY FOR USE AS PICKUP IN ENERGY HARVESTING}

\begin{abstract}
In recent years, the term energy harvest has been used in a large class of applications whose main function is the recovery of energy available from sources, such as solar, thermal, mechanical, among others, who otherwise would be wasted. In this sense, the objective of this study is the development and characterization of a core of iron powder obtained by mechanical alloying, as well as using this core as a pickup in a process of induction energy harvesting in order to observe their ability to harvest energy. The iron was characterized by x-ray diffraction and scanning electron microscopy and conducted bench tests to evaluate the amount of energy harvested from
\end{abstract}

the pickup developed. Structural analysis showed that the powder obtained is single phase, without presence of impurities. The micrographs are homogeneous characteristic, with low porosity. Magnetic characteristics of the material obtained from the classification as soft ferromagnetic material, due to the narrow hysteresis loop. In tests for energy harvesting, the core presented satisfactory results, because it was able to provide a maximum level of $0.41 \mathrm{~mW}$ power when subjected to an electric current in your primary winding of the 15 .

KEYWORDS: Iron, energy harvesting, magnetic induction, powder metallurgy. 


\section{INTRODUÇÃO}

Colheita de energia é o termo utilizado para descrever a transformação de energia ambiente não utilizável (vibracional, eólica, térmica, solar) em energia elétrica utilizável (BELHORA et al., 2012). Existem dispositivos capazes de aproveitar as mais diversas fontes de energia presentes no ambiente e transformá-las em energia elétrica, para alimentar aparelhos eletrônicos de baixo consumo de energia. Além das mais tradicionais células solares e geradores eólicos, a colheita de energia aproveita também o calor e as vibrações presentes no ambiente, entre outras (BANDYOPADHYAY e CHANDRAKASAN, 2012). Atualmente, as teorias, experiências e demonstrações encontradas na colheita de energia são várias e podem ser integrada em diversas áreas, tais como, medicina, meios de transporte, meios de comunicação, eletrônica, aplicações militares (FERNANDES, 2012).

Como outro tipo de fonte alternativa, os campos eletromagnéticos estão se tornando uma fonte viável para colheita de energia, embora não seja uma fonte natural de energia disponível, pois ocorre em torno de linhas de distribuição de energia existentes, tem se pesquisado como uma fonte alternativa para aplicações em colheita de energia (energy harvesting). De acordo com a Lei de Faraday, se um ímã móvel atravessar uma bobina fixa é possível obter uma variação de fluxo magnético, induzindo desta forma uma corrente elétrica (SORDIASHIE, 2012). Dispositivos para colheita de energia que converte energia do ambiente por indução magnética em energia elétrica têm atraído muito interesse em diversos setores.

No momento em que se pretende desenvolver a aplicação para gerar energia elétrica a partir do efeito eletromagnético, é necessário considerar que os dispositivos responsáveis por este efeito, tendem a ter dimensões um pouco maiores quando comparados a outros componentes geradores, uma vez que estes requerem o uso de bobinas.

Para resolver este problema, a pesquisa sobre materiais utilizados na confecção de dispositivos para converter a energia do ambiente, tais como a energia solar (SUN, et al. 2014), a energia vibracional (CHIRIAC et al., 2012), energia térmica (AMOUYAL, 2013) ou a eletromagnética (BIBO et al., 2012) em energia elétrica tem sido intensivamente realizada. Na fabricação de núcleos magnéticos moles (elevada permeabilidade magnética) adequados, é essencial a obtenção de materiais com propriedades magnéticas superiores (satisfatórias). Hoje em dia, pesquisas são feitas para se desenvolver outros tipos de materiais que tenham essa propriedade ainda mais acentuada, possibilitando a uma maior quantidade de aplicações.

As propriedades elétricas e magnéticas dependem da microestrutura final do material que pode ser controlada por diversos parâmetros físico-químicos, tais como processamento utilizado e natureza química dos componentes.

Um dos materiais que possui características magnéticas atrativas é o ferro, bem como algumas de suas ligas. $O$ ferro apresenta elevada permeabilidade, ciclo de histerese estreito e baixa resistividade, esta combinação de propriedades faz deste um material apropriado para aplicação em circuitos magnéticos.

Ferro e componentes de aço foram produzidos comercialmente por metalurgia do pó a muitos anos e os desenvolvimentos na tecnologia do pó têm sido impressionantes. Hoje, o desempenho dos componentes fabricados a partir de métodos metalúrgicos do pó é 
favoravelmente comparado com aqueles feitos a partir de técnicas convencionais de forjamento. A compactação de pós tornou-se mais fácil, produzindo produtos de alta densidade e de alto desempenho. $O$ progresso também tem sido feito na obtenção de melhores tolerâncias dimensionais após a sinterização dos compactados (SRINIVASA, 2002).

Com base neste enfoque, este trabalho tem como objetivo o desenvolvimento de um núcleo magnético de ferro puro por meio da técnica de mecanosíntese (metalurgia do pó), e sua caracterização estrutural e magnética bem como investigar a utilização do núcleo magnético de ferro obtido em colheita de energia (Energy Harvesting) por indução magnética.

\section{METODOLOGIA}

\subsection{Síntese}

A liga de ferro foi desenvolvida utilizando os princípios de mecanosíntese, definida como um processo de síntese no estado sólido a temperatura moderada, por moagem, em geral a seco, de mistura de pós de elementos puros ou combinados, num moinho a alta energia (BENJAMIN, 1970). O ferro puro utilizado para confecção do núcleo (marca Aldrich com 99,999\% de pureza) foi processado por moagem em um moinho de bolas planetário, com rotações de $300 \mathrm{rpm}$ e tempo de moagem de 5 horas. A compactação foi realizada a temperatura ambiente, com cargas 700 $\mathrm{MPa}$, em prensa hidráulica manual, de ação simples. O núcleo produzido foi sinterizado a temperatura de $1150^{\circ} \mathrm{C}$ por 1 hora. A taxa de aquecimento aplicada foi de $10^{\circ} \mathrm{C} / \mathrm{min}$ e de resfriamento de $20^{\circ} \mathrm{C} / \mathrm{min}$. Utilizou-se um forno resistivo da marca Nabertherm, percorrido por uma corrente contínua de gás hélio e na presença de titânio, estes dois recursos foram utilizados visando uma proteção contra oxidação superficial do material.

\subsection{Caracterização estrutural}

A caracterização microestrutural foi realizada por difração de raios $X$ sendo possível observar as fases presentes, para tanto foi utilizado o difratômetro de raios-X SIEMENS D5000, sendo empregado a radiação CuK $\alpha$, cujo comprimento de onda é $\lambda=1,5406 \AA ̊$. A identificação das fases foi realizada utilizando o banco de dados JCPDS. A análise morfológica do ferro foi realizada em um microscópio eletrônico de varredura em um microscópio SHIMADZU modelo SSX-550.

\subsection{Teste de bancada}

Após a obtenção do núcleo de ferro, o mesmo foi bobinado com fio de cobre esmaltado de seção 21 AWG adquirindo em seu enrolamento primário e secundário um número de 198 e 50 voltas respectivamente para o teste de bancada. Para a realização dos experimentos de colheita de energia, utilizou-se uma plataforma de testes, em que o núcleo, denominado de $P E$, foi submetido a um nível de corrente máxima de $15 \mathrm{~A}$ no terminal primário, com o intuito de observar os níveis de potência obtidos nas cargas compreendidas entre $10 \Omega$ e $10 \mathrm{~K} \Omega$ conectadas ao terminal secundário.

Para a determinação das características magnéticas foram realizados testes em vazio, utilizando a plataforma experimental, aplicando-se níveis de correntes entre OA a 292 A no terminal primário. Para a determinação da curva de magnetização e da permeabilidade magnética, foram colhidas quarenta e duas amostras de dados pelo osciloscópio em um intervalo de tensão 
de entrada de $1 \mathrm{~V}$ de pico. Toda a modelagem matemática do processo foi desenvolvida objetivando a determinação do laço B x H (laço de histerese), para a obtenção dos parâmetros magnéticos: curva de magnetização, densidade de fluxo magnético de saturação, permeabilidade do material, permeabilidade relativa, densidade de fluxo residual e força coerciva. O processo de colheita de energia e caracterização dos parâmetros magnéticos foi analisado com o auxílio de um osciloscópio por intermédio da aferição de dados dos níveis de corrente e tensão adquiridos na carga e no terminal primário do núcleo. Esses dados foram processados via software SCILAB, por meio de programação desenvolvida, que consiste na analise dos valores de corrente e tensão para a determinação dos parâmetros magnéticos citados.

\section{RESULTADOS E DISCUSSÃO}

Os resultados são descritos em duas etapas conforme a metodologia realizada, em que a primeira etapa se refere a caracterização estrutural do núcleo obtido. E a segunda é referente ao teste de bancada realizado para colheita de energia do núcleo obtido e caracterização magnética.

\subsection{Caracterização estrutural}

Na Figura 1 está representado o difratograma de raios $X$ da amostra de ferro puro obtido por mecanosíntese.

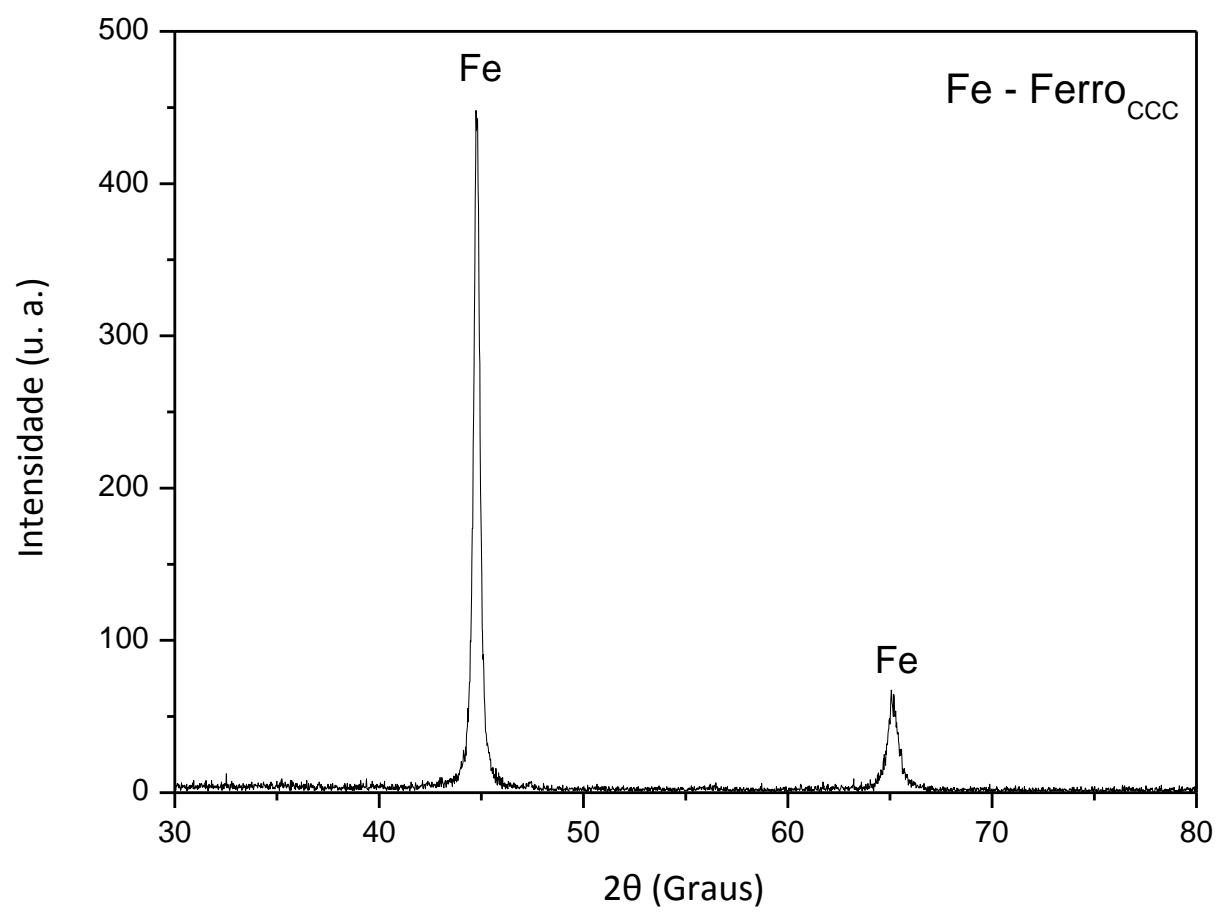

Figura 1: Difratograma de raios $X$ do ferro obtido por mecanosíntese (moagem de alta energia).

$O$ resultado de difração de raios $X$ revela que o produto obtido é de alta pureza sem presença de fases secundárias provenientes de contaminação adquiridas durante o processo de moagem. De acordo com o difratograma apresentado na Figura 1, pode-se observar que a amostra é cristalina com picos característicos do ferro CCC (cúbico de corpo centrado), de acordo com a ficha cristalográfica JCPDS (\#87-0721), grupo espacial $\operatorname{Im} \overline{3} m$, com parâmetro de rede de aproximadamente $a=2,87 \AA ̊$. 
Este mesmo padrão de difração foi observado por Kumar e Selvarajan (2008), que avaliaram o ferro puro obtido por spray plasma, adquiriram amostras em que o padrão de difração de raios $X$ mostra os picos característicos de Fe $(110)$ e Fe $(200)$ em $44,93^{\circ}$ e $65,49^{\circ}$, respectivamente. Porém nessa amostra foram detectados picos de intensidade relativamente baixa correspondente ao óxido de ferro, mas não identificado. Os pós são vistos como sendo de natureza policristalina devido ao método de processamento.

Na Figura 2, encontram-se as micrografias obtidas por microscopia eletrônica de varredura (MEV) da amostra do ferro puro após sua obtenção e sinterização.
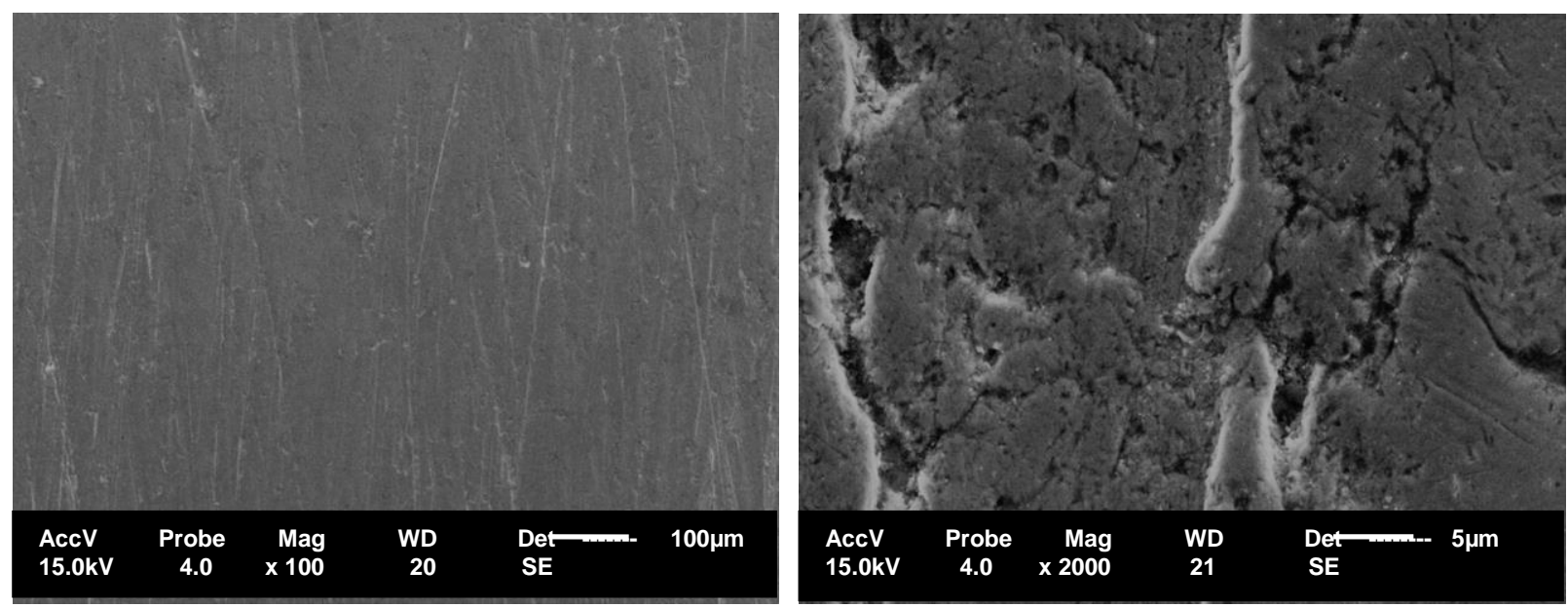

Figura 2: Micrografia do ferro puro após obtenção e sinterização com aumentos (a) 100x e (b) 2000x.

Pode-se observar que a amostra possui superfície rugosa com presença de ranhuras, é notada a presença de porosidade intergranular e defeitos provenientes do processo de compactação da amostra. Este resultado está de acordo com os resultados apresentados por Shaibanie Ghambari (2011), que avaliaram as propriedades metalúrgicas, geométricas e mecânicas, bem como a tensão residual dos pós de ferro produzidos pelo método "target jet milling" e comparados com pós produzidos por moagem com bolas convencional. Os resultados mostraram que o ferro produzido em moagem convencional e compactado a $500 \mathrm{Mpa}$ apresentam falha quase completamente intergranular e a existência grandes poros entre as partículas, segundo os autores isso ocorre devido à baixa pressão de compactação.

\subsection{Caracterização magnética}

Os parâmetros magnéticos do núcleo foram obtidos a partir do laço de histerese, Figura 3 (a). Nesse laço, foram extraídos valores de $B$ (densidade de fluxo magnético) e $H$ (intensidade de campo magnético) para a obtenção da curva de magnetização, Figura 3(b). A permeabilidade relativa é calculada na taxa de variação da densidade de campo magnético com relação a variação da intensidade de campo magnético.

Os gráficos da Figura 3 foram obtidos com variação de corrente de 0A a 292A. 


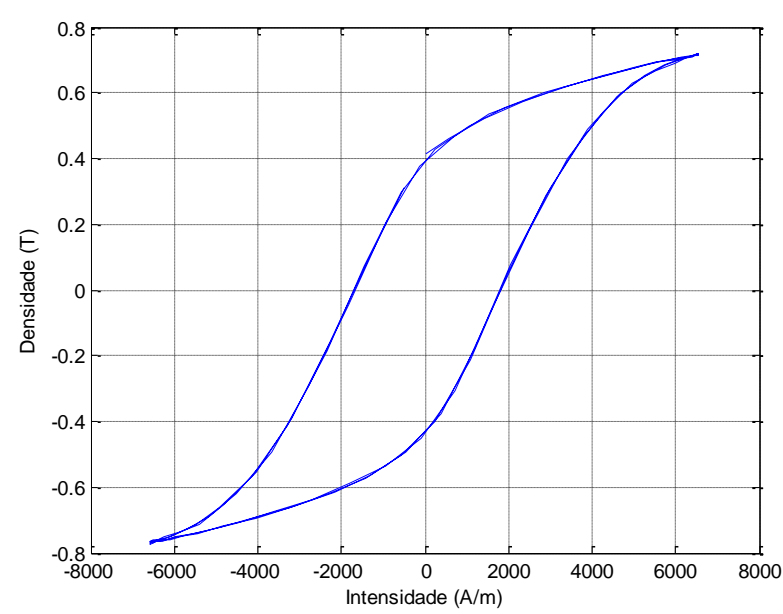

(a)

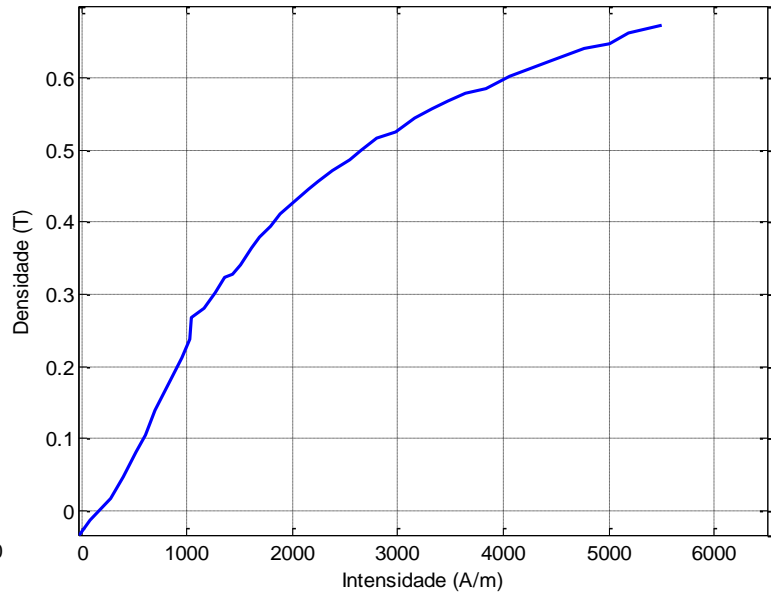

(b)

Figura 3: (a) Laço B x H (b) Curva de magnetização

Observando-se o laço B $\times$ H foi possível obter a densidade de saturação, a densidade residual, que são os valores referentes da diferença da origem do sinal ao valor em que a curva intercepta o eixo B para valores positivos e negativos, e a força coerciva, que são os valores referentes da diferença da origem do sinal ao valor em que a curva intercepta o eixo $\mathrm{H}$ para valores negativos e positivos.

De acordo com os dados colhidos da Figura 3, foi possível obter os seguintes parâmetros:

Tabela 1: Resultados experimentais das características magnéticas

\begin{tabular}{c|c|c|c|c|c}
\hline$\mu_{r}$ Máximo & $\begin{array}{c}\mu_{m} \\
{[\mathbf{H} / \mathbf{m}]}\end{array}$ & $\begin{array}{c}H_{c} \\
{[\mathbf{A} / \mathbf{m}]}\end{array}$ & $\begin{array}{c}\text { Bsat } \\
\text { Inicial } \\
{[\mathbf{T}]}\end{array}$ & $\begin{array}{c}\mathrm{Br} \\
{[\mathbf{T}]}\end{array}$ & $\begin{array}{c}\text { RMS } \\
\text { RM] }\end{array}$ \\
\hline 100 & 0,00012 & 1700 & 0,6 & 0,4 & 292 \\
\hline
\end{tabular}

Em que $\mu_{r}$ é a permeabilidade relativa, $\mu_{m}$ permeabilidade do material, $H_{c}$ a força coerciva, $B_{r}$ o fluxo residual, $B_{s a t}$ a densidade de fluxo de saturação e $I_{p}$ a corrente do primário.

Para a obtenção da densidade de fluxo de saturação, fez-se necessário atribuir uma corrente de 292A no terminal primário do núcleo, devido núcleo apresentar uma baixa permeabilidade magnética relativa. De acordo com o gráfico da Figura 3a, é possível observar que o núcleo possui consideráveis perdas por histerese.

\subsection{Colheita de Energia}

Para colheita de energia foi realizado ensaios, em que se verificou a potência obtida em função da carga, Figura 4. É possível visualizar os valores de potência obtidos para o captador confeccionado com ferro puro obtido por mecanosíntese. Embora os ensaios tenham sido realizados para valores entre $10 \Omega$ e $100 \Omega$. Os maiores níveis de potência estão localizados entre $10 \Omega$ e $30 \Omega$ de carga, para valores de próximos $100 \Omega$, a potência medida no captador tende a diminuir alcançando valores menores que $0,2 \mathrm{~mW}$. Foi obtido um valor máximo de potência de $0,41 \mathrm{~mW}$ para uma carga conectada ao terminal secundário de $10 \Omega$. 


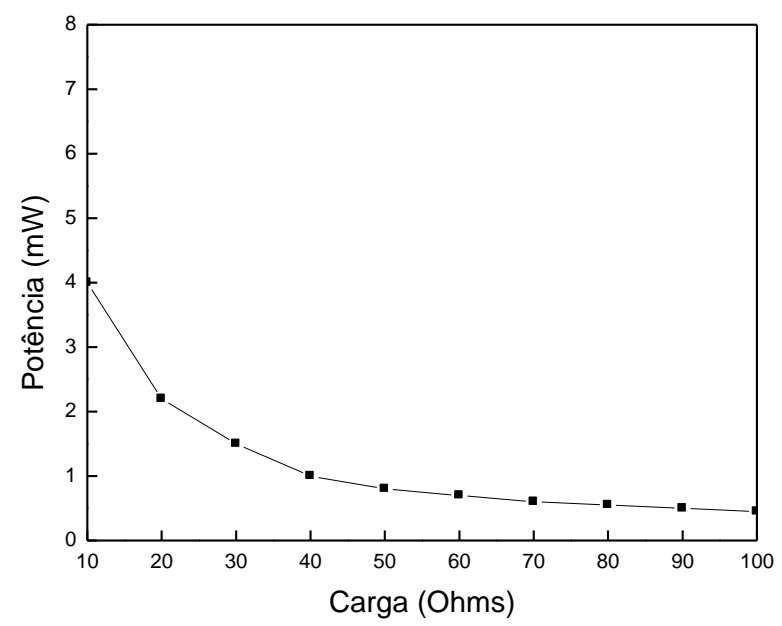

Figura 4: Potência X carga do captador de energia.

\section{CONCLUSÃO}

A forma de síntese utilizada para a obtenção do ferro foi apropriada visto a obtenção de material puro, ou seja, monofásico e parâmetros semelhantes aos teóricos. Foi constatado que o núcleo obteve ótimos resultados de captação de fluxo magnético, uma boa permeabilidade magnética relativa e baixos níveis de densidade de fluxo residual. Em contrapartida, o núcleo apresentou um alto valor de força coerciva compreendida em $1700 \mathrm{~A} / \mathrm{m}$ e perdas por histerese consideráveis. Em suma, o núcleo teve um bom comportamento quando no processo de captação de campo magnético, consequentemente resultando em boas características magnéticas. Para o processo de captação de energia foi possível obter um nível de potência de 0,41 $\mathrm{mW}$ em uma carga de $10 \Omega$ com uma corrente no primário de 15A. Sendo assim, sua aplicabilidade pode estar voltada para captação de energia em linhas de potência visando alimentação de dispositivos de baixo consumo.

\section{AGRADECIMENTOS}

Os autores agradecem à CAPES e ao CNPq pelo apoio financeiro para realização desta pesquisa.

\section{REFERÊNCIAS BIBLIOGRÁFICAS}

1. AMOUYAL, Y. On the role of lanthanum substitution defects in reducing lattice thermal conductivity of the $\mathrm{AgSbTe}_{2}(\mathrm{P} 4 / \mathrm{mmm})$ thermoelectric compound for energy conversion applications. Computational Materials Science, v. 78, p. 98-103, 2013.

2. BANDYOPADHYAY, S., CHANDRAKASAN, A. P. Platform Architecture for Solar, Thermal, and Vibration Energy Combining With MPPT and Single Inductor. IEEE Journal of Solid-State Circuits, v. 47, p. 2199-2215, 2012. doi: 10.1109/JSSC.2012.2197239

3. BELHORA, F., COTTINET, P.-J., GUYOMAR, D., LEBRUN, L., HAJJAJI, A., MAZROUI, M., BOUGHALEB, Y. Hybridization of electrostrictive polymers and electrets for mechanical energy harvesting. Sensors and Actuators A, v. 183, p. 50- 56, jun. 2012. 
4. BENJAMIN, J. S. Dispersion Strengthened Superalloys by Mechanical Alloying. Metallurgical Transactions, v. 1, n. 10, p. 2943-2951, 1970.

5. BIBO, A., MASANA, R., KING, A., LI, G., DAQAQ, M. F. Electromagnetic ferrofluid-based energy harvester. Physics Letters A, v. 376, p. 2163-2166, 2012.

6. CHAVEZ-GONZALEZ, A. F., AGUILA-MUNOZ, J., PEREZ-BENITEZ, J. A., ESPINA-HERNANDEZ, J. H. Finite differences software for the numeric analysis of a non-destructive electromagnetic testing system, In: International Conference on Electronics, Communications and Computers (CONIELECOMP), p. 82-86, March 2013.

7. CHIRIAC, H., IBU, M., LUPU, N., SKORVANEK, I., ÓVÁRI, T.-A. Nanocrystalline ribbons for energy harvesting applications. Journal of Applied Physics, v. 115, 2014. doi: 10.1063/1.4864437.

8. FERNANDES, C. S. Microgeradores Elétricos. Minho, 2012. Dissertação de Mestrado - Escola de Engenharia - Universidade do Minho, 2012.

9. KUMAR, S., SELVARAJAN, V. Plasma spheroidization of iron powders in a non-transferred DC thermal plasma jet. Materials Characterization, v. 59 p. $781-785,2008$.

10. SHAIBANI, M. E., GHAMBARI, M. Characterization and comparison of gray cast iron powder produced by target jetmilling and high energy ball milling of machining scraps. Powder Technology, v. 212, p. 278-283, 2011.

11. SORDIASHIE, E. Electromagnetic Harvesting to Power Energy Management Sensors in the Built Environment. Nebraska, 2012. Dissertations and Student Research - Architectural Engineering - University of Nebraska-Lincoln, 2012.

12. SRINIVASAN, N. S. Dynamic study of changes in structure and morphology during the heating and sintering of iron powder. Powder Technology, v. 124, p. 40-44, 2002.

13. SUN, S.-S., BROOKS, J., NGUYEN, T., HARDING, A., WANG, D., DAVID, T. Novel Organic and Polymeric Materials for Solar Energy Conversions. Energy Procedia, v. 57, p. 79 - 88, 2014.

14. WANG, J., YIN, H., QU, X. Analysis of density and mechanical properties of high velocity compacted iron powder. Acta Metallurgica Sinica (English Letters), v. 22, n.6, p. 447-453, 2009. 